HUPD-9220

December 1992

\title{
Dynamical CP Violation in Composite Higgs Models
}

\author{
S. Hashimoto, T T. Inagaki and T. Muta \\ Department of Physics, Hiroshima University \\ Higashi-Hiroshima, Hiroshima 724, Japan
}

\begin{abstract}
The dynamical origin of the $\mathrm{CP}$ violation in electroweak theory is investigated in composite Higgs models. The mechanism of the spontaneous CP violation proposed in other context by Dashen is adopted to construct simple models of the dynamical $\mathrm{CP}$ violation. Within the models the size of the neutron electric dipole moment is estimated and the constraint on the $\varepsilon$-parameter in K-meson decays is discussed.
\end{abstract}

PACS numbers: 11.30.Er, 11.30.Qc, 12.15.Cc

\footnotetext{
${ }^{*}$ Supported in part by Monbusho Grant-in-Aid under the contract No.040011

${ }^{\dagger}$ Supported in part by Monbusho Grant-in-Aid for Scientific Research (C) under the contract No. 04640301
} 


\section{INTRODUCTION}

The $\mathrm{CP}$ violation is described by phases appearing in the Kobayashi-Maskawa matrix [1] in the standard theory of quarks and leptons. The $\mathrm{CP}$ violating phases are introduced only when the number of the quark-lepton generations is equal to or greater than three. In other words the reason why we have the $\mathrm{CP}$ violation in nature is that we have three generations of quarks and leptons. The $\mathrm{CP}$ violating phases are partially determined by experimental data in the neutral K-meson system. The prediction for the neutron electric dipole moment [2] based on the Kobayashi-Maskawa $\mathrm{CP}$ violating phases (KM phases) is extremely small and is well below the experimental lower bound[3]. Thus the standard theory with Kobayashi-Maskawa CP violation is consistent with the present experimental situation.

The KM phases are introduced as free parameters in the standard theory. From the point of view of the fundamental theory of quarks and leptons this situation is not satisfactory and we would like to see where is the theoretical origin of the KM phases describing the $\mathrm{CP}$ violation.

One of the possibilities to explain the KM phases by the more fundamental origin is to introduce the complex vacuum expectation values for the Higgs field as discussed by Weinberg more than a decade ago[团. In this approach it is required to have at least three Higgs doublets in order to interprete the full KM phases. This mechanism suggests that the spontaneous electroweak symmetry breaking has something to do with the origin of the $\mathrm{CP}$ violation.

Pushing forward this idea we are naturally led to the composite Higgs models where the Higgs field is replaced by a composite system of fundamental fermions. There are a variety of the composite Higgs models including the technicolor model [5], top-condensation model[6, 7], fourth-generation model[ [8] and color-sextet quark model[9]. In the composite Higgs models the $\mathrm{CP}$ violation may occur if the complex vacuum expectation value would result for the composite field $\bar{\psi} \psi$ with fundamental fermion $\psi$. The realization of such circumstance has once been suggested long time ago by Dashen in other context [10].

The idea of Dashen will be recapitulated in the next section and will be applied straightforwardly to the composite Higgs models. Eichten, Lane and Preskill 11] have adopted Dashen's idea in the technicolor model to elucidate the mechanism of the dynamical CP violation. In their paper the general framework of generating the dynamical CP violation was presented and some physical consequences were pointed 
out. Later Goldstein[12] has reconsidered the problem and constructed a model of the dynamical $\mathrm{CP}$ violation with two quark and techniquark doublets. This model, however, fails to give rise to the $\mathrm{CP}$-violating phase unless one introduces extra leptons or one assumes an existence of the strong $\mathrm{CP}$ violation in the technicolor sector.

In the present paper we would like to construct some simple examples of the dynamical $\mathrm{CP}$ violation in the composite Higgs models. In our models we assume the presence of two flavors of up(down)-type extra fundamental quarks and three flavors of up(down)-type ordinary quarks. We start with the Lagrangian with flavor symmetry (i.e. all fermions massless) in which a nonvanishing vacuum expectation value develops for the composite field $\bar{\psi} \psi$ with $\psi$ the fundamental fermion. To this Lagrangian we add flavor-symmetry breaking terms to realize the quark mass hierarchy. We consider transformations which mixes the flavors of quarks. We find a special solution for the transformations which gives the true vacuum with the proper direction. According to this special solution the $\mathrm{CP}$ violating terms are generated in the flavor-symmetry breaking part of the Lagrangian.

The main purpose of our argument is to show the usefulness of the Dashen mechanism for the dynamical $\mathrm{CP}$ violation in a transparent way. Our model is too simple to explain the KM phases practically and should be elaborated to reproduce the standard theory as a low-energy effective theory. If our model has something to do with the nature, it has to be consistent with the existing experimental observations. Thus we calculate the contribution in our model to the electric dipole moment of the neutron and the $\varepsilon$-parameter in $\mathrm{K}$ decays. Both quantities are found to be consistent with the experimental data if the cut-off $\Lambda$ existing in the model is larger than $800 \mathrm{TeV}$ which is consistent with the cut-off set by the FCNC restriction[13].

It should be remarked that any model of the spontaneous $\mathrm{CP}$ violation suffers from the cosmological domain wall problem. In the present paper we are interested in constructing simple examples of the dynamical $\mathrm{CP}$ violation and we tentatively circumvent the problem by assuming that the dynamical $\mathrm{CP}$ violation takes place before the inflation period. 


\section{DASHEN MECHANISM IN COMPOSITE HIGGS MODELS}

Here in the present section we briefly review the Dashen mechanism of the spontaneous $\mathrm{CP}$ violation with the application to the composite Higgs models.

We start with the Lagrangian $\mathcal{L}_{0}$ symmetric under the flavor group

$$
G_{F}=\prod_{\rho} U_{V}\left(n_{\rho}\right) \otimes U_{A}\left(n_{\rho}\right)
$$

where $n_{\rho}$ is the number of quark flavors belonging to the irreducible representation $\rho$ in the underlying gauge group and $U_{V}\left(U_{A}\right)$ is the unitary group associated with the vector (axialvector) currents. Here by the term "quark" we mean the ordinary quarks as well as the fermions needed to generate the composite Higgs field. The quark fields included in the Lagrangian $\mathcal{L}_{0}$ are all massless to guarantee the underlying gauge symmetry and the flavor symmetry.

We assume that the flavor symmetry $G_{F}$ is broken dynamically by the presence of the nonvanishing vacuum expectation value for the composite field made of certain quark fields,

$$
\langle\bar{\psi} \psi\rangle \neq 0
$$

Here we have chosen the vacuum for which

$$
\left\langle\bar{\psi} \gamma_{5} \psi\right\rangle=0
$$

The quarks acquire masses according to the dynamical breaking of the flavor symmetry $G_{F}$. The remaining flavor symmetry if any will be denoted by $S_{F}$. As is wellknown the vacuum satisfying Eqs.(2.2) and (2.3) is not unique and thus we have degenerate vacua in $G_{F} / S_{F}$. These degenerate vacua point to arbitrary direction in $G_{F} / S_{F}$.

Now we add to $\mathcal{L}_{0}$ the term $\mathcal{L}^{\prime}$ which explicitly breaks the flavor symmetry $G_{F}$. We assume that $\mathcal{L}^{\prime}$ is $\mathrm{CP}$-invariant and $S_{F}$-symmetric. The degeneracy of the vacua mentioned above is now resolved in the system described by the total Lagrangian

$$
\mathcal{L}=\mathcal{L}_{0}+\mathcal{L}^{\prime}
$$

The direction of the vacuum thus determined, however, does not necessarily guarantee the conditions (2.2) and (2.3). Hence we need to make a transformation on the field to recover the conditions

$$
\psi^{\prime}=U \psi
$$


with $U$ the transformation belonging to $G_{F}$. By this transformation the form of the symmetry breaking term $\mathcal{L}^{\prime}$ will be modified so that $\mathrm{CP}$ violating terms in general show up in $\mathcal{L}^{\prime}$. We will call this mechanism of the spontaneous CP violation 10 the Dashen mechanism. In the following we would like to apply the Dashen mechanism to the case of the composite Higgs models.

In electroweak theory the Higgs fields are introduced as elementary scalar fields. Accordingly the Higgs mass, Higgs self-coupling constant and Higgs-fermion Yukawa-coupling constants are all arbitrary parameters. In the composite Higgs model the Higgs particle appears as a composite system of some fundamental fermions and some of the parameters in the standard electroweak theory are predictable in principle. The Lagrangian corresponding to this model may be given by

$$
\mathcal{L}_{0}=\mathcal{L}_{Q C D}+\mathcal{L}_{E W}+\mathcal{L}_{D Y N}
$$

where $\mathcal{L}_{Q C D}$ is the ordinary QCD Lagrangian for quarks, $\mathcal{L}_{E W}$ is the electroweak Lagrangian without Higgs fields and $\mathcal{L}_{D Y N}$ is the dynamical term which is assumed to be responsible for generating the composite Higgs system as a bound state (this term may be thought of as a low-energy effective Lagrangian stemming from the more fundamental Lagrangian).

The Higgs particle appears as a bound state of the fundamental fermions $\psi$ and the bound state is assumed to generate a condensation,

$$
\langle\bar{\psi} \psi\rangle \neq 0 .
$$

The fundamental fermions as well as the ordinary quarks acquire a mass according to the condensation. The mass of the fundamental fermions should be of the order of the weak scale in order to guarantee that the resulting effective theory be the standard electroweak theory.

In the technicolor model [5] the fundamental fermion is the techniquark, in the top-condensation model[6] it is the top quark with mass close to the weak scale, in the fourth-generation model [8] it is the heavy quark in the assumed fourth generation and in the color-sextet model[9] it is the quark in the sextet representation of the color $\mathrm{SU}(3)$.

In the following we would like to present simple models of the dynamical $\mathrm{CP}$ violation in the composite Higgs models. 


\section{SIMPLE MODELS OF DYNAMICAL CP VIOLATION}

\section{A. General formalism}

Here we first present a general argument in constructing simple models of the dynamical CP violation in the composite Higgs models. We consider $n_{\rho}$ flavors of fundamental quarks in the representation $\rho$ of the color $\mathrm{SU}(3)$ or other symmetry group (we call this symmetry governing the fundamental quarks the symmetry $\mathrm{S}$ ) and $n_{3}$ flavors of ordinary quarks in the triplet representation of the color $\mathrm{SU}(3)$. The fundamental quarks may or may not have a color degree of freedom.

We will discuss transformations which mix the flavors of the fundamental and ordinary quarks among themselves. Since this transformation has to conserve charges, the mixing occurs only among the up-type (or down-type) fundamental and ordinary quarks. For simplicity we consider only up-type fundamental and ordinary quarks.

According to Goldstein's analysis [12] one finds that only two flavors of the fundamental and ordinary quarks are not sufficient to realize the Dashen mechanism. Hence we try a model with 2 flavors of the up-type fundamental quarks $Q$ and 3 flavors of the up-type ordinary quarks $q$ :

$$
Q=(U, C), \quad q=(u, c, t)
$$

We assume that $Q$ belongs to the N-plet of the fundamental symmetry $\mathrm{S}$ and $q$ belongs to the color triplet. It is understood that our model equally applies to the system of the down-type quarks

$$
Q=(D, S), \quad q=(d, s, b)
$$

In the following by the term "quark" we generically mean both fundamental and ordinary quarks.

As a $G_{F}$ breaking Hamiltonian density $\mathcal{H}^{\prime}$ we take the following four-fermion terms

$$
\begin{aligned}
\mathcal{H}^{\prime}=-\mathcal{L}^{\prime}= & G^{Q}{ }_{\alpha \beta} \bar{Q}_{L} \tau^{\alpha} Q_{R} \bar{Q}_{R} \tau^{\beta} Q_{L} \\
+ & G^{Q q}{ }_{\alpha \beta} \bar{Q}_{L} \tau^{\alpha} Q_{R} \bar{q}_{R} \lambda^{\beta} q_{L}+\text { h.c. } \\
+ & G^{q}{ }_{\alpha \beta} \bar{q}_{L} \lambda^{\alpha} q_{R} \bar{q}_{R} \lambda^{\beta} q_{L}
\end{aligned}
$$


where $G^{Q}{ }_{\alpha \beta}, G^{Q q}{ }_{\alpha \beta}$ and $G^{q}{ }_{\alpha \beta}$ are coupling parameters among fundamental quarks $Q$ and ordinary quarks $q$ which depend on indices $\alpha$ and $\beta$ of the flavor $\mathrm{SU}(2)$ and $\mathrm{SU}(3)$ matrices $\tau^{\alpha}(\alpha=1,2,3)$ and $\lambda^{\alpha}(\alpha=1,2, \ldots, 8)$ respectively. In Eq.(3.3) the fundamental symmetry indices and color indices are suppressed and are understood to be contracted between adjoining quarks. There would be other possibilities of contracting these indices. We, however, confine ourselves to the case of Eq.(3.3).

We require the CP invariance and hermiticity of the Lagrangian (3.3). We then have

$$
\begin{aligned}
& G^{Q}{ }_{\alpha \beta} \tau^{\alpha}{ }_{r r^{\prime}} \tau^{\beta}{ }_{s s^{\prime}}=G^{Q}{ }_{\beta \alpha} \tau^{\alpha}{ }_{r^{\prime} r} \tau^{\beta}{ }_{s^{\prime} s}=\left(G^{Q}{ }_{\beta \alpha} \tau^{\alpha}{ }_{r^{\prime} r} \tau^{\beta}{ }_{s^{\prime} s}\right)^{*}, \\
& G^{Q q}{ }_{\alpha \beta} \tau^{\alpha}{ }_{r r^{\prime}} \lambda^{\beta}{ }_{s s^{\prime}}=\left(G^{Q q}{ }_{\alpha \beta} \tau^{\alpha}{ }_{r r^{\prime}} \lambda^{\beta}{ }_{s s^{\prime}}\right)^{*} \text {, } \\
& G^{q}{ }_{\alpha \beta} \lambda^{\alpha}{ }_{r r^{\prime}} \lambda^{\beta}{ }_{s s^{\prime}}=G^{q}{ }_{\beta \alpha} \lambda^{\alpha}{ }_{r^{\prime} r} \lambda^{\beta}{ }_{s^{\prime} s}=\left(G^{q}{ }_{\beta \alpha} \lambda^{\alpha}{ }_{r^{\prime} r} \lambda^{\beta}{ }_{s^{\prime} s}\right)^{*},
\end{aligned}
$$

where indices $r, r^{\prime}, s, s^{\prime}$ represent flavors of $Q$ and $q$, i.e. $U, C, u, c, t$.

Our first task is to find the correct vacuum under the Lagrangian

$$
\mathcal{L}=\mathcal{L}_{0}+\mathcal{L}^{\prime}
$$

where $\mathcal{L}_{0}$ is the Lagrangian given by Eq.(2.6) and $\mathcal{L}^{\prime}$ is given by Eq.(3.3). Let us denote by $|\overline{0}\rangle$ the ground state (vacuum) for a system governed by the Lagrangian (3.5) and by $|0\rangle$ the ground state for $\mathcal{L}_{0}$ which is invariant under $S_{F}$. To find the ground state $|\overline{0}\rangle$ we try to minimize the energy

$$
E(W)=\left\langle\overline{0}\left|\mathcal{H}^{\prime}\right| \overline{0}\right\rangle=\left\langle 0\left|W^{\dagger} \mathcal{H}^{\prime} W\right| 0\right\rangle
$$

by suitably choosing the transformation $W$ in $G_{F}$. The transformation $W$ is induced by the transformation $U$ of fermion fields $Q$ and $q$ :

$$
Q_{L, R}^{\prime}=U_{L, R}^{Q} Q_{L, R}, \quad q_{L, R}^{\prime}=U_{L, R}^{q} q_{L, R}
$$

where $U_{L, R}^{Q}$ is the transformation belonging to the left-handed(right-handed) flavor $\mathrm{SU}(2)$ for fundamental quarks $Q$ and $U_{L, R}^{q}$ belonging to the $\mathrm{SU}(3)$ for ordinary quarks $q$. The transformation $W$ is a function of these fermion transformations:

$$
W=W(U)
$$

where we represent generically by $U$ the transformations $U_{L, R}^{Q}$ and $U_{L, R}^{q}$. We find 


$$
\begin{aligned}
E(W)= & G^{Q}{ }_{\alpha \beta}\left\langle 0\left|\bar{Q}_{L} U_{L}^{Q_{L}^{\dagger}} \tau^{\alpha} U_{R}^{Q} Q_{R} \bar{Q}_{R} U_{R}^{Q^{\dagger}} \tau^{\beta} U^{Q}{ }_{L} Q_{L}\right| 0\right\rangle \\
& +G^{Q q}{ }_{\alpha \beta}\langle 0| \bar{Q}_{L} U^{Q^{\dagger}} \tau^{\alpha} U^{Q}{ }_{R} Q_{R} \bar{q}_{R} U^{q \dagger}{ }_{R}^{\dagger} \lambda^{\beta} U^{q}{ }_{L} q_{L}+\text { h.c. }|0\rangle \\
& +G^{q}{ }_{\alpha \beta}\left\langle 0\left|\bar{q}_{L} U_{L}^{q \dagger} \lambda^{\alpha} U^{q}{ }_{R} q_{R} \bar{q}_{R} U_{R}^{q \dagger} \lambda^{\beta} U^{q}{ }_{L} q_{L}\right| 0\right\rangle .
\end{aligned}
$$

Since the state $|0\rangle$ is invariant under $S_{F}$, we may express the following amplitudes as given below:

$$
\begin{aligned}
& \left\langle 0\left|\bar{Q}_{L r} Q_{R r^{\prime}}\right| 0\right\rangle=\Delta^{Q}{ }_{0} \delta_{r r^{\prime}},\left\langle 0\left|\bar{q}_{L r} q_{R r^{\prime}}\right| 0\right\rangle=\Delta^{q}{ }_{0} \delta_{r r^{\prime}}, \\
& \left\langle 0\left|\bar{Q}_{L r} Q_{R r^{\prime}} \bar{Q}_{R s} Q_{L s^{\prime}}\right| 0\right\rangle=\Delta^{Q} \delta_{r r^{\prime}} \delta_{s s^{\prime}}+\Delta^{\prime Q} \delta_{r s^{\prime}} \delta r^{\prime} s, \\
& \left\langle 0\left|\bar{Q}_{L r} Q_{R r^{\prime}} \bar{q}_{R s} q_{L s^{\prime}}\right| 0\right\rangle=\Delta^{Q q} \delta_{r r^{\prime}} \delta_{s s^{\prime}}, \\
& \left\langle 0\left|\bar{q}_{L r} q_{R r^{\prime}} \bar{q}_{R s} q_{L s^{\prime}}\right| 0\right\rangle=\Delta^{q} \delta_{r r^{\prime}} \delta_{s s^{\prime}}+\Delta^{\prime q} \delta_{r s^{\prime}} \delta r^{\prime} s
\end{aligned}
$$

where parameters $\Delta$ are chosen to be real. After some algebra we obtain

$$
\begin{aligned}
E(W)= & g^{Q}{ }_{\alpha \beta} \operatorname{Tr}\left[U^{Q} \tau^{\alpha}\right] \operatorname{Tr}\left[\tau^{\beta} U^{Q \dagger}\right] \\
& +r g^{Q q}{ }_{\alpha \beta} \operatorname{Tr}\left[U^{Q} \tau^{\alpha}\right] \operatorname{Tr}\left[\lambda^{\beta} U^{{ }^{\dagger}}\right]+\text { h.c. } \\
& +r^{2} g^{q}{ }_{\alpha \beta} \operatorname{Tr}\left[U^{q} \lambda^{\alpha}\right] \operatorname{Tr}\left[\lambda^{\beta} U^{{ }^{\dagger \dagger}}\right],
\end{aligned}
$$

where matrices $U^{Q}$ and $U^{q}$ and parameters $g^{Q}{ }_{\alpha \beta}, g^{Q q}{ }_{\alpha \beta}, g^{q}{ }_{\alpha \beta}$ and $r$ are given by the following relations:

$$
\begin{gathered}
U^{Q}=U_{R}^{Q} U_{L}^{Q^{\dagger}}, \quad U^{q}=U_{R}^{q} U_{L}^{q \dagger}, \\
g_{\alpha \beta}^{Q}=G_{\alpha \beta}^{Q} \Delta^{Q}, r g_{\alpha \beta}^{Q q}=G_{\alpha \beta}^{Q q} \Delta^{Q q}, r^{2} g_{\alpha \beta}^{q}=G_{\alpha \beta}^{q} \Delta^{q}, \\
r=\frac{\langle\bar{q} q\rangle}{\langle\bar{Q} Q\rangle} .
\end{gathered}
$$

Here we introduced parameter $r$ in order to show explicitly the relative size of the three kinds of parameters $G^{Q}{ }_{\alpha \beta}, G^{Q q}{ }_{\alpha \beta}$ and $G^{q}{ }_{\alpha \beta}$. The parameter $r$ is the ratio of the ordinary and fundamental mass scale [5 and its size is assumed to be

$$
r \sim\left(\frac{1 \mathrm{GeV}}{1 \mathrm{TeV}}\right)^{3}=10^{-9}
$$

Our task is to minimize $E(W)$ given in Eq.(3.11) by changing $U$ and find the solution for $U$. With $U$ determined in this procedure we rewrite $\mathcal{L}^{\prime}$ to see whether $\mathrm{CP}$ violating terms are generated in $\mathcal{L}^{\prime}$. 


\section{B. Special solutions}

We would like to find the general solution for $U$ to minimize $E(W)$ in Eq.(3.11). It is, however, quite complicated to obtain the general solution and we shall confine ourselves to some special solutions to this problem.

We first consider the following specialization,

$$
\begin{aligned}
& g^{Q}{ }_{00}=g^{Q}{ }_{33}\left(\equiv g^{Q}\right)<0, \\
& 3 g^{Q q}{ }_{00}=-\sqrt{3} g^{Q q}{ }_{08}=\sqrt{3} g^{Q q}{ }_{30}=2 g^{Q q}{ }_{38}\left(\equiv g^{Q q}\right)>0, \\
& g^{Q}{ }_{\alpha \beta}=0, g^{Q q}{ }_{\alpha \beta}=0: \text { otherwise. }
\end{aligned}
$$

Parametrizing the matrix elements of matrices $U^{Q}$ and $U^{q}$ by

$$
\begin{aligned}
& U^{Q}{ }_{i j}=u^{Q}{ }_{i j} \exp \left(i \theta^{Q}{ }_{i j}\right) \quad \text { with } i, j=1,2 \\
& U^{q}{ }_{i j}=u^{q}{ }_{i j} \exp \left(i \theta^{q}{ }_{i j}\right) \quad \text { with } i, j=1,2,3
\end{aligned}
$$

where $u$ 's and $\theta$ 's are real constants constrained by the unitality of $U$, we obtain

$$
\begin{aligned}
& E(W)=2 g^{Q}\left\{\left(u^{Q} 11\right)^{2}+\left(u^{Q}{ }_{22}\right)^{2}\right\} \\
&+2 r g^{Q q}\left\{\frac{\sqrt{3}}{2} u^{Q}{ }_{11} u^{q}{ }_{11} \cos \left(\theta^{Q} 11-\theta^{q}{ }_{11}\right)\right. \\
&+\frac{\sqrt{3}}{2} u^{Q}{ }_{11} u^{q}{ }_{22} \cos \left(\theta^{Q}{ }_{11}-\theta^{q}{ }_{22}\right) \\
&+u^{Q}{ }_{11} u^{q}{ }_{33} \cos \left(\theta^{Q}{ }_{11}-\theta^{q} 33\right) \\
&-\frac{\sqrt{3}}{2} u^{Q}{ }_{22} u^{q}{ }_{11} \cos \left(\theta^{Q}{ }_{22}-\theta^{q} 11\right) \\
&-\frac{\sqrt{3}}{2} u^{Q}{ }_{22} u^{q} 22 \cos \left(\theta^{Q}{ }_{22}-\theta^{q} 22\right) \\
&\left.+u^{Q}{ }_{22} u^{q} 33 \cos \left(\theta^{Q}{ }_{22}-\theta^{q} 33\right)\right\} \\
&+\mathrm{O}\left(r^{2}\right) .
\end{aligned}
$$

In deriving Eq.(3.18) we kept only the terms up to the first order of the small number

$r$. We expand parameters $u$ 's and $\theta$ 's in powers of $r$ and look for the minimum of the energy $E(W)$ to the first order of $r$ :

$$
\begin{aligned}
& E=E^{0}+E^{1} r+\mathrm{O}\left(r^{2}\right), \\
& u=u^{0}+u^{1} r+\mathrm{O}\left(r^{2}\right), \\
& \theta=\theta^{0}+\theta^{1} r+\mathrm{O}\left(r^{2}\right),
\end{aligned}
$$


where we have omitted the suffices $i$ and $j$ and the superfix $Q$ or $q$ in the parameters $u$ and $\theta$. After some algebra we find

$$
\begin{aligned}
& E^{0}=2 g^{Q}\left\{\left(u^{Q 0}{ }_{11}\right)^{2}+\left(u^{Q 0}{ }_{22}\right)^{2}\right\}, \\
& E^{1}=4 g^{Q}\left(u^{Q 0}{ }_{11} u^{Q 1}{ }_{11}+u^{Q 0}{ }_{22} u^{Q 1}{ }_{22}\right) \\
& +2 g^{Q q}\left\{\frac{\sqrt{3}}{2} u^{Q 0}{ }_{11} u^{q 0}{ }_{11} \cos \left(\theta^{Q 0}{ }_{11}-\theta^{q 0}{ }_{11}\right)\right. \\
& +\frac{\sqrt{3}}{2} u^{Q 0}{ }_{11} u^{q 0}{ }_{22} \cos \left(\theta^{Q 0}{ }_{11}-\theta^{q 0}{ }_{22}\right) \\
& +u^{Q 0}{ }_{11} u^{q 0}{ }_{33} \cos \left(\theta^{Q 0}{ }_{11}-\theta^{q 0}{ }_{33}\right) \\
& -\frac{\sqrt{3}}{2} u^{Q 0}{ }_{22} u^{q 0}{ }_{11} \cos \left(\theta^{Q 0}{ }_{22}-\theta^{q 0}{ }_{11}\right) \\
& -\frac{\sqrt{3}}{2} u^{Q 0}{ }_{22} u^{q 0}{ }_{22} \cos \left(\theta^{Q 0}{ }_{22}-\theta^{q 0}{ }_{22}\right) \\
& \left.+u^{Q 0}{ }_{22} u^{q 0}{ }_{33} \cos \left(\theta^{Q 0}{ }_{22}-\theta^{q 0}{ }_{33}\right)\right\} \text {. }
\end{aligned}
$$

Since we chose $g^{Q}<0, u^{Q 0}{ }_{11}$ and $u^{Q 0}{ }_{22}$ may be taken to be unity according to Eq.(3.20). We find the following set of parameters $u$ 's and $\theta$ 's to minimize $E^{1}$.

$$
\begin{array}{ll}
u^{Q 0}{ }_{11} & =u^{Q 0}{ }_{22}=1, \\
u^{Q 1}{ }_{11} & =u^{Q 1}{ }_{22}=0, \\
u^{q 0}{ }_{11}=u^{q 0}{ }_{22}=u^{q 0}{ }_{33}=1, & (\bmod 2 \pi) \\
\theta^{Q 0}{ }_{11}=\theta \pm \frac{\pi}{3}, \theta^{Q 0}{ }_{22}=\theta \mp \frac{\pi}{3}, & \\
\theta^{q 0}{ }_{11}=\theta \mp \frac{\pi}{2}, \theta^{q 0}{ }_{22}=\theta \mp \frac{\pi}{2}, \theta^{q 0}{ }_{33}=\theta \pm \pi, & (\bmod 2 \pi)
\end{array}
$$

where $\theta$ is the free parameter. Thus the transformation matrices $U^{Q}$ and $U^{q}$ are given by

$$
\begin{aligned}
U^{Q} & =U_{R}^{Q} U_{L}^{Q^{\dagger}}=e^{i \theta}\left(\begin{array}{cc}
e^{ \pm i \frac{\pi}{3}} & 0 \\
0 & e^{\mp i \frac{\pi}{3}}
\end{array}\right) \\
U^{q} & =U_{R}^{q} U_{L}^{q_{L}^{\dagger}}=e^{i \theta}\left(\begin{array}{ccc}
e^{\mp i \frac{\pi}{2}} & 0 & 0 \\
0 & e^{\mp i \frac{\pi}{2}} & 0 \\
0 & 0 & e^{ \pm i \pi}
\end{array}\right) .
\end{aligned}
$$

In the present paper we would like to construct a model without the strong $\mathrm{CP}$ violation and so we set

$$
\theta=0
$$


We redefine the fields $Q$ and $q$ in such a way that

$$
\begin{aligned}
& Q_{L}^{\prime}=Q_{L}, \quad Q_{R}^{\prime}=U_{L}^{Q} U_{R}^{Q^{\dagger}} Q_{R}, \\
& q_{L}^{\prime}=q_{L}, \quad q_{R}^{\prime}=U^{q}{ }_{L} U_{R}^{q \dagger} q_{R} .
\end{aligned}
$$

so that the following condition is recovered,

$$
\begin{aligned}
& \left\langle 0\left|\bar{Q}^{\prime}{ }_{L r} Q_{R r^{\prime}}^{\prime}\right| 0\right\rangle=\Delta^{Q}{ }_{0} \delta_{r r^{\prime}}, \\
& \left\langle 0\left|\bar{q}_{L r}^{\prime}{ }_{L r} q_{R r^{\prime}}^{\prime}\right| 0\right\rangle=\Delta^{q}{ }_{0} \delta_{r r^{\prime}} .
\end{aligned}
$$

Expressed by the new fields $Q^{\prime}$ and $q^{\prime}$ the Hamiltonian density $\mathcal{H}^{\prime}$ takes the form

$$
\begin{aligned}
& \mathcal{H}^{\prime}=-\mathcal{L}^{\prime}=G^{Q}{ }_{\alpha \beta} \bar{Q}^{\prime}{ }_{L} \tau^{\alpha} U^{Q}{ }_{R} U^{Q^{\dagger}}{ }_{L}^{\prime} Q_{R}^{\prime} \bar{Q}_{R}^{\prime} U^{Q}{ }_{L} U^{Q^{\dagger}} \tau^{\beta} Q_{L}^{\prime} \\
& +G^{Q q}{ }_{\alpha \beta} \bar{Q}^{\prime}{ }_{L} \tau^{\alpha} U^{Q}{ }_{R} U^{Q^{\dagger}}{ }_{L}^{\prime} Q_{R}^{\prime} \bar{q}_{R}{ }_{R} U^{q}{ }_{L} U^{q \dagger}{ }_{R}^{\beta} q_{L}^{\prime}+\text { h.c. } \\
& +G^{q}{ }_{\alpha \beta} \bar{q}^{\prime}{ }_{L} \lambda^{\alpha} U^{q}{ }_{R} U^{q \dagger}{ }_{L}^{\prime} \bar{q}_{R} \bar{q}_{R} U^{q}{ }_{L} U^{q \dagger}{ }_{R} \lambda^{\beta} q_{L}^{\prime} \text {. }
\end{aligned}
$$

We find in Eq.(3.27) that the second and third term in general violate $\mathrm{CP}$ since these two terms can not be made real. To see this situation more explicitly we rewrite Eq.(3.27) in the following form,

$$
\begin{aligned}
& \mathcal{H}^{\prime}=2 G^{Q}\left(\bar{U}_{L}^{\prime} U_{R}^{\prime} \bar{U}^{\prime}{ }_{R} U_{L}^{\prime}+\bar{C}^{\prime}{ }_{L} C_{R}^{\prime} \bar{C}^{\prime}{ }_{R} C_{L}^{\prime}\right) \\
& +G^{Q q}\left[\frac{\sqrt{3}}{2}\left(e^{i \frac{5}{6} \pi} \bar{U}_{L}^{\prime} U_{R}^{\prime}-e^{i \frac{1}{6} \pi} \bar{C}_{L}^{\prime} C_{R}^{\prime}\right)\left(\bar{u}_{R}^{\prime} u_{L}^{\prime}+\bar{c}_{R}^{\prime} c_{L}^{\prime}\right)\right. \\
& \left.+\left(e^{-i \frac{2}{3} \pi} \bar{U}^{\prime}{ }_{L} U_{R}^{\prime}+e^{-i \frac{4}{3} \pi} \bar{C}^{\prime}{ }_{L} C_{R}^{\prime}\right)\left(\bar{t}_{R}{ }_{R} t_{L}^{\prime}\right)\right]+ \text { h.c. } \\
& +\frac{4}{3} G^{q} 88 \bar{t}_{L}^{\prime} t_{R}^{\prime} \bar{t}^{\prime}{ }_{R} t_{L}^{\prime}+\cdots \\
& +\left(G^{q}{ }_{44}-G^{q}{ }_{55}\right) e^{i \frac{3}{2} \pi} \bar{u}_{L}^{\prime} t_{R}^{\prime} \bar{u}_{R}^{\prime} t_{L}^{\prime}+\cdots \text {. }
\end{aligned}
$$

We now clearly observe that the new expression of the Hamiltonian density $\mathcal{H}^{\prime}$ includes $\mathrm{CP}$ violating terms. In fact the second and fourth term in $\mathcal{H}^{\prime}$ apparently violate $\mathrm{CP}$. On the other hand the other terms do not violate $\mathrm{CP}$.

It may be interesting to see the form of the up-type quark mass matrix $\left(M_{i j}\right)$ in the present specific model. After some algebra we find

$$
\left(M_{i j}\right)=2 g^{Q q}\left(\begin{array}{ccc}
-3 / 4 & 0 & 0 \\
0 & -3 / 4 & 0 \\
0 & 0 & -1 / 2
\end{array}\right) .
$$

Obviously the form (3.29) does not properly reproduce the quark mass hierarchy and so our model is not realistic. 
We next consider the following specific choice of our coupling parameters,

$$
\begin{aligned}
& g^{Q}{ }_{00}=g^{Q}{ }_{33}\left(\equiv g^{Q}\right)<0, \\
& \sqrt{3} g^{Q q}{ }_{00}=-g^{Q q}{ }_{08}=\sqrt{3} g^{Q q}{ }_{30}=2 g^{Q q}{ }_{38}\left(\equiv g^{Q q}\right)>0, \\
& g^{Q}{ }_{\alpha \beta}=0, g^{Q q}{ }_{\alpha \beta}=0: \text { otherwise. }
\end{aligned}
$$

Following the same procedure as before we find the solution

$$
\begin{aligned}
U^{Q} & =U_{R}^{Q} U_{L}^{Q^{\dagger}}=e^{i \theta}\left(\begin{array}{cc}
e^{ \pm i \frac{\pi}{4}} & 0 \\
0 & e^{\mp i \frac{\pi}{4}}
\end{array}\right), \\
U^{q} & =U_{R}^{q} U_{L}^{q_{L}^{\dagger}}=e^{i \theta}\left(\begin{array}{ccc}
e^{\mp i \frac{\pi}{2}} & 0 & 0 \\
0 & e^{\mp i \frac{\pi}{2}} & 0 \\
0 & 0 & e^{ \pm i \pi}
\end{array}\right) .
\end{aligned}
$$

The up-type quark mass matrix corresponding to the above solution reads

$$
\left(M_{i j}\right)=2 g^{Q q}\left(\begin{array}{ccc}
-\sqrt{6} / 4 & 0 & 0 \\
0 & -\sqrt{6} / 4 & 0 \\
0 & 0 & -\sqrt{6} / 2
\end{array}\right) .
$$

We observe that in this case the top quark is heavier than the up and charm quark.

\section{Models}

We found the $\mathrm{CP}$ violating interaction Lagrangian as a result of special solutions of the minimum $E(W)$ condition. Thus we succeeded in constructing the simple model of the dynamical $\mathrm{CP}$ violation. In deriving the model we made some simplifying assumptions. This simplification made the model far from explaining the real situation in standard theory. For example our model Hamiltonian does not reproduce the KM matrix correctly. In order to get the full KM matrix we have to relax our assumptions and minimize $E(W)$ with the full expression of the transformation matrix $U$ (We have to abolish the assumption that $U$ be a diagonal matrix). This attempt will be made in a separate work. We are, however, interested in estimating physical effects in low-energy phenomena which are predicted by the Hamiltonian. Such estimation may help examining whether our model serves as a prototype of the real theory of the dynamical CP violation for standard theory.

The system of quarks we assumed consists of the up-type 2-flavor fundamental quarks $Q$ and 3-flavor ordinary quarks $q$ as shown in Eq.(3.1). We have not yet specified the symmetry group $\mathrm{S}$ to which the fundamental quarks $Q$ belong.

A natural possibility is to identify the symmetry group $\mathrm{S}$ to the technicolor $\mathrm{SU}(\mathrm{N})$. In this case the fundamental quark $Q$ is the techniquark[5] belonging to the 
$\mathrm{N}$-dimensional fundamental representation of the technicolor $\mathrm{SU}(\mathrm{N})$.

Another possibility is to identify the symmetry group $\mathrm{S}$ to the color $\mathrm{SU}(3)$. In this case the fundamental quark $Q$ is the color-sextet quark [9] belonging to the 6-dimensional representation of the color $\mathrm{SU}(3)$.

These two possibilities fit the previous argument quite well and constitute two practical models of the dynamical $\mathrm{CP}$ violation.

It is also possible to identify the fundamental quarks $Q$ to the top quark in the top-condensation model[6] (or in the top-color model[]]). In this case, however, we are not able to get the nontrivial $\mathrm{CP}$ violating phase within our framework

Yet another possibility is to identify the fundamental fermion $Q$ to the quark in the assumed fourth generation [8]. In this case again it is impossible to obtain the nontrivial $\mathrm{CP}$ violating phase in our approach.

In the following application we are in mind the technicolor model as well as the color-sextet quark model.

\section{LOW ENERGY EFFECTS}

In our simple model introduced in the last section the KM matrix is real and diagonal. This is because we have taken a paticular choice for a $G_{F}$ breaking Lagrngian $\mathcal{L}^{\prime}$ and have neglected the higher order terms in $r$. Starting with the more general assumption we could have obtained the KM matrix with off-diagonal elements and complex phases.

In the present section we consider possible low-energy effects originating from the model Lagrangian (3.27). By this analysis we will be able to compare the lowenergy CP-violating effects of dynamical origin with the one in the standard origin of the CP violation (i.e. through the KM phase).

Our Hamiltonian reads

$$
H=H_{0}+H_{\text {cons }}^{\prime}+H_{\text {viol }}^{\prime}
$$

where $H_{0}$ is the Hamiltonian derived from Lagrangian $\mathcal{L}_{0}, H_{\text {cons }}^{\prime}$ is the CP conserving part of the Hamiltonian defined by integrating Eq.(3.27) over the space variables and $H_{\text {viol }}^{\prime}$ is the CP violating part. In the following we consider two typical low-energy 
effects derived from the Hamiltonian (4.1).

\section{A. Neutron electric dipole moment}

Since the Lagrangian $\mathcal{L}^{\prime}$ includes the energy scale at which the four-fermion interactions are induced from the more fundamental gauge theory, it is expected that our estimate of the neutron electric dipole moment depend on this energy scale. This means that this fundamental energy scale, i.e. the cut-off parameter $\Lambda$, may be constrained by the experimental information on the neutron electric dipole moment.

We estimate the size of the contribution to the neutron electric dipole moment coming from our CP-violating Lagrangian $\mathcal{L}^{\prime}$ given in Eq.(3.27).

The neutron electric dipole moment $d_{n}$ is given in terms of the quark dipole moments $d_{u}$ and $d_{d}$ in the naive quark model such that

$$
d_{n}=\frac{4 d_{d}-d_{u}}{3}
$$

The electric dipole moment of quarks is calculated through the following term in the quark electromagnetic form factor at zero momentum transfer,

$$
-d_{q} \bar{u} \sigma_{\mu \nu} \gamma_{5} q^{\nu} u
$$

where suffix $q$ represents the $\mathrm{u}$ or $\mathrm{d}$ quark and $q^{\nu}$ is the momentum transfer for quarks (momentum carried by the virtual photon) and $u$ is the Dirac spinor for quark $q$.

We start with the Lagrangian $\mathcal{L}^{\prime}$ given in Eq.(3.27). For the later calculational convenience we introduce auxiliary fields $\phi$ and use the following effective Lagrangian instead of the four-fermion type Lagrangian (3.27):

$$
\mathcal{L}^{\prime}=-\bar{\psi}\left(\phi^{\dagger} \frac{1-\gamma_{5}}{2}+\phi \frac{1+\gamma_{5}}{2}\right) \psi+G^{-1} \phi^{\dagger} \phi .
$$

The use of the above auxiliary-field Lagrangian makes it easier to classify the relevant Feynman diagrams contributing to the quark electric dipole moment and to perform the higher-order loop calculations.

At one-loop level diagrams shown in Fig.1 contribute to the quark electromagnetic form factor. 
Fig.1a

Fig.1b

Fig.1 One-loop diagrams for the electromagnetic vertex function of quarks represented by the use of auxiliary field $\phi$.

As is easily seen the diagram in Fig.1a has no tensor structure corresponding to the electric dipole moment. The contribution of Fig.1b to the electric dipole moment is found to vanish. Thus there is no one-loop contribution to the quark electric dipole moment.

We next examine the two-loop contribution to the quark electric dipole moment. The relevant diagrams are shown in Fig.2.

Fig.2a

Fig. 2b

Fig.2c

Fig.2 Two-loop diagrams for the electromagnetic vertex function of quarks.

The Feynman amplitudes corresponding to these diagrams are in general quartically divergent. The quartically divergent part of the amplitudes, however, has no tensor structure of the electric dipole moment and hence the leading contribution of these diagrams to the quark electric dipole moment is quadratically divergent. As is seen by direct calculations, the diagrams in Figs.2a and $2 b$ have no quadratically divergent contribution to the quark electric dipole moment. The reason for this is that the helicity of the quark flips three times in these diagrams. Accordingly the leading quadratic divergence exists only in the diagram in Fig.2c. In the following we will 
calculate the quadratically divergent part of the Feynman amplitude corresponding to the diagram in Fig.2c.

The Feynman amplitude $F$ corresponding to the diagram in Fig.2c reads

$$
\begin{aligned}
& F=\sum_{i, j, k} \int \frac{d^{4} p}{(2 \pi)^{4} i} \frac{d^{4} p^{\prime}}{(2 \pi)^{4} i}\left[G_{j i u k}^{q} G_{k j i u}^{q}\right. \\
& \times\left\{\frac{1+\gamma_{5}}{2} \frac{1}{m_{i}-\not p_{1}+\not p^{\prime}} \frac{1-\gamma_{5}}{2} \frac{1}{m_{j}-\not p_{1}+\not p+\not p^{\prime}}\right. \\
& \times \frac{1-\gamma_{5}}{2} \frac{1}{m_{k}-\not \phi_{1}+\not p}\left(Q_{k} e \gamma_{\mu}\right) \frac{1}{m_{k}-\not p_{2}+\not p} \frac{1+\gamma_{5}}{2} \\
& +\frac{1+\gamma_{5}}{2} \frac{1}{m_{i}-\not p_{1}+\not p^{\prime}} \frac{1-\gamma_{5}}{2} \frac{1}{m_{j}-\not p_{1}+\not p+\not p^{\prime}}\left(Q_{j} e \gamma_{\mu}\right) \\
& \times \frac{1}{m_{j}-\not p_{2}+\not p+\not p^{\prime}} \frac{1-\gamma_{5}}{2} \frac{1}{m_{k}-\not p_{2}+\not p} \frac{1+\gamma_{5}}{2} \\
& +\frac{1+\gamma_{5}}{2} \frac{1}{m_{i}-\not p_{1}+\not p^{\prime}}\left(Q_{i} e \gamma_{\mu}\right) \frac{1}{m_{j}-\not p_{2}+\not p^{\prime}} \frac{1-\gamma_{5}}{2} \\
& \left.\times \frac{1}{m_{j}-\not p_{2}+\not p+\not p^{\prime}} \frac{1-\gamma_{5}}{2} \frac{1}{m_{k}-\not p_{2}+\not p} \frac{1+\gamma_{5}}{2}\right\} \\
& \left.+G_{u k j i}^{q} G_{i u k j}^{q}\left\{\gamma_{5} \rightarrow-\gamma_{5}\right\}\right],
\end{aligned}
$$

where $p_{1}\left(p_{2}\right)$ is the momentum of the incoming (outgoing) quark. Here in Eq.(4.5) the charge $Q_{j}$ is equal to $2 / 3$ for up-type quarks, i.e. $j=u, c, t$, and is equal to $-1 / 3$ for down-type quarks, i.e. $j=d, s, b$. By extracting the quadratically divergent part $F^{\text {div }}$ of Eq.(4.5) we obtain

$$
F^{d i v}=\frac{2 \Lambda^{2}}{(4 \pi)^{4}} \sum_{i, j, k} Q e \operatorname{Im}\left\{G_{j i u k}^{q} G_{k j i u}^{q}\right\} m_{j}\left(i A_{j} k_{\mu} \gamma_{5}-B_{j} \sigma_{\mu \nu} q^{\nu} \gamma_{5}\right),
$$

where $A_{j}$ and $B_{j}$ are given by

$$
\begin{aligned}
A_{j}=2\left(\ln \frac{\Lambda^{2}}{m_{j}^{2}}-1\right) & \\
+\int_{0}^{1} d x \int_{0}^{1} d y \int_{0}^{1-y} d z[ & \left\{\frac{4}{x}(2-3 y)+(3-5 y)\right\} \ln \left|\frac{x(1-x)+1-y-z}{1-y-z}\right| \\
& \quad-\frac{3 x(1-x)(1-2 y)}{x(1-x)+1-y-z} \\
& +\frac{1}{2} \frac{x(1-x)}{\{x(1-x)+1-y-z\}^{2}}\left\{x(1-x)\left(3(3-7 y)-\frac{8}{x}(2-3 y)\right)\right. \\
B_{j}=\int_{0}^{1} d x \int_{0}^{1} d y \int_{0}^{1-y} d z \frac{1}{x}\left(4 \ln \left|\frac{x(1-x)+1-y-z}{1-y-z}\right|\right. & \left.\quad-\frac{x(1-x)\{4 x(1-x)+3(1-y-z)\}}{\{x(1-x)+1-y-z\}^{2}}\right) .
\end{aligned}
$$


After some algebra we derive the following formula for the quadratically divergent part of the electric dipole moment of the up-quark $d_{u}$

$$
d_{u}=\frac{2}{3} e \frac{\Lambda^{2}}{(4 \pi)^{4}} \sum_{i, j, k} \operatorname{Im}\left\{G_{j i u k}^{q} G_{k j i u}^{q}\right\} m_{j}\left(A_{j}+B_{j}\right) .
$$

Performing the integration in Eq.(4.7) we finally find the explicit expression for the quadratically divergent part of the up-quark electric dipole moment,

$$
d_{u}=\frac{2}{3} e \frac{2 \Lambda^{2}}{(4 \pi)^{4}} \sum_{i, j, k} \operatorname{Im}\left\{G_{j i u k}^{q} G_{k j i u}^{q}\right\} m_{j}\left[2 \ln \frac{\Lambda^{2}}{m_{j}^{2}}-2.57\right] .
$$

Apparently the dominant contribution in the above formula to the up-quark dipole moment comes from the top-quark intermediate state. Keeping only the top-quark contribution to Eq.(4.9) and taking into accout that

$$
\operatorname{Im}\left\{G_{j i u k}^{q} G_{k j i u}^{q}\right\} \sim \frac{g^{4}}{4 \Lambda^{4}} \sim \frac{(2 \pi)^{2}}{\Lambda^{4}},
$$

we find

$$
d_{u}=e \frac{m_{t}}{48 \pi^{2} \Lambda^{2}}\left[4 \ln \frac{\Lambda}{m_{t}}-2.57\right] .
$$

Since $d_{u} \gg d_{d}$, we find that $d_{n}=d_{u} / 3$. Assuming that $m_{t}=140 \mathrm{GeV}$ and taking into account the experimental upper bound of the neutron electric dipole moment [3] we realize that the effective cut-off of the loop integral should satisfy

$$
\Lambda>800 \mathrm{TeV} .
$$

The above lower bound for the cut-off $\Lambda$ is of the same order as the one set by the FCNC restriction [13]. If we use the value of $\Lambda$ set by the FCNC restriction which will be described in Eq.(4.22) and calculate $d_{n}$ through Eq.(4.11), we find $d_{n} \sim$ $5 \times 10^{-27} \mathrm{ecm}$. This prediction is surely much smaller than the present experimental bound. In the standard model with the KM phase the neutron electric dipole moment is calculated and is found to be extremely small[2]. Our result (4.11) and (4.12) gurantees this property of the standard model.

\section{B. K-meson system}

The only known experimental information on the $\mathrm{CP}$ violation exists in the $\mathrm{K}$-meson decays. In this subsection we discuss the $\varepsilon$-parameter which is determined by measuring the charge asymmetry in the semileptonic decay of the $\mathrm{K}^{0}$ meson and 
the $2 \pi$ decay of the $K_{L}^{0}$ meson.

$\mathrm{K}^{0}$-meson states $\left|\mathrm{K}_{L}^{0}\right\rangle$ and $\left|\mathrm{K}_{S}^{0}\right\rangle$ are defined as

$$
\begin{gathered}
\left|\mathrm{K}_{L}^{0}\right\rangle=\frac{1}{\sqrt{2\left(1+|\varepsilon|^{2}\right)}}\left\{(1+\varepsilon)\left|\mathrm{K}^{0}\right\rangle+(1-\varepsilon)\left|\overline{\mathrm{K}}^{0}\right\rangle\right\} \\
\left|\mathrm{K}_{S}^{0}\right\rangle=\frac{1}{\sqrt{2\left(1+|\varepsilon|^{2}\right)}}\left\{(1+\varepsilon)\left|\mathrm{K}^{0}\right\rangle-(1-\varepsilon)\left|\overline{\mathrm{K}}^{0}\right\rangle\right\}, \\
\left(\left|\mathrm{K}^{0}\right\rangle=-\mathrm{CP}\left|\overline{\mathrm{K}}^{0}\right\rangle\right),
\end{gathered}
$$

With the non-vanishing $\varepsilon$ the K-meson mass eigenstates are different from the eigenstates of CP. We have

$$
\varepsilon=\frac{\left\langle\mathrm{K}^{0}|H| \overline{\mathrm{K}}^{0}\right\rangle^{\frac{1}{2}}-\left\langle\overline{\mathrm{K}}^{0}|H| \mathrm{K}^{0}\right\rangle^{\frac{1}{2}}}{\left\langle\mathrm{~K}^{0}|H| \overline{\mathrm{K}}^{0}\right\rangle^{\frac{1}{2}}+\left\langle\overline{\mathrm{K}}^{0}|H| \mathrm{K}^{0}\right\rangle^{\frac{1}{2}}} \simeq \frac{\left\langle\mathrm{K}^{0}\left|H_{\text {viol }}^{\prime}\right| \overline{\mathrm{K}}^{0}\right\rangle}{\left\langle\mathrm{K}^{0}\left|\left(H_{0}+H_{\text {cons }}^{\prime}\right)\right| \overline{\mathrm{K}}^{0}\right\rangle} .
$$

Here we require that

$$
\left|\left\langle\mathrm{K}^{0}\left|\left(H_{0}+H_{\text {cons }}^{\prime}\right)\right| \overline{\mathrm{K}}^{0}\right\rangle\right| \gg\left|\left\langle\mathrm{K}^{0}\left|H_{\text {viol }}^{\prime}\right| \overline{\mathrm{K}}^{0}\right\rangle\right|
$$

The Hamiltonian $H_{v i o l}^{\prime}$ contains the following term,

$$
i \operatorname{Im}(G) \int d^{3} x \bar{s}_{L} d_{R} \bar{s}_{R} d_{L}+\text { h.c. },
$$

where $G$ is the corresponding four-fermion coupling constant and s and d represent the s and d-quark fields. Although in our model $\operatorname{Im}(G)$ vanishes, we here consider the more general cace in which $\operatorname{Im}(G) \neq 0$. Using the PCAC relation (It should be remembered that a specific choice of the contraction of color indices is made in Eq.(3.3) ), we find

$$
\left\langle\mathrm{K}^{0}\left|\bar{s}_{L} d_{R} \bar{s}_{R} d_{L}\right| \overline{\mathrm{K}}^{0}\right\rangle=-\frac{B_{K}(\mu) f_{K}^{2} m_{K}^{4}}{2\left(m_{s}+m_{d}\right)^{2}},
$$

where $B_{K}(\mu)$ is the so-called B-parameter, $f_{K}$ is the K-meson decay constant and $m_{K}$, $m_{d}$ and $m_{s}$ are the mass of the K-meson, d-quark and s-quark respectively. After some calculation, we obtain

$$
\left\langle\mathrm{K}^{0}\left|H_{v i o l}^{\prime}\right| \overline{\mathrm{K}}^{0}\right\rangle \simeq-i \frac{\operatorname{Im}(G) B_{K}(\mu) f_{K}^{2} m_{K}^{3}}{4\left(m_{d}+m_{s}\right)^{2}}\left\langle\mathrm{~K}^{0} \mid \mathrm{K}^{0}\right\rangle .
$$

We see by definition

$$
\left\langle\mathrm{K}^{0}\left|\left(H_{0}+H_{\text {cons }}^{\prime}\right)\right| \overline{\mathrm{K}}^{0}\right\rangle \simeq \frac{1}{2}\left(\Delta M-\frac{i}{2} \Delta \Gamma\right)\left\langle\mathrm{K}^{0} \mid \mathrm{K}^{0}\right\rangle,
$$

where $\Delta M$ and $\Delta \Gamma$ are the $\mathrm{K}_{L}-\mathrm{K}_{S}$ difference of the mass and decay width respectively. Accordingly we obtain

$$
\varepsilon \simeq \frac{-i \operatorname{Im}(G) B_{K}(\mu) f_{K}^{2} m_{K}^{3}}{2\left(\Delta M-\frac{i}{2} \Delta \Gamma\right)\left(m_{d}+m_{s}\right)^{2}} .
$$


By inserting experimental data in Eq.(4.20) we find

$$
\operatorname{Im}(G) \sim 10^{-9} \mathrm{TeV}^{-2}
$$

The above result (4.21) is about $10^{2}$ times smaller than that obtained by the FCNC restriction 13 ,

$$
\operatorname{Re}(G)<10^{-7} \mathrm{TeV}^{-2}
$$

\section{CONCLUSION}

Applying Dashen's mechanism to the composite Higgs models we succeeded to find simple models of the dynamical CP violation. Although our models have to be further elaborated to explain the actual KM phase, they represent an essential ingredient of the dynamical CP violation in the standard model and may be thought of as prototype models which accommodate the $\mathrm{CP}$ violation in the standard model.

In order to see whether our model could be in conformity with experimental situations we examined low-energy consequences of our model. By estimating the $\varepsilon$ parameter in the neutral K-meson decays and the neutron electric dipole moment we derived the lower bound on the cut-off parameter using the available experimental informations. The cut-off parameter signals, at the scale determined by the lowenergy data, the existence of the deeper theory for which our model is an effective theory. The lower bound we obtained is consistent with the one required by the constraint on the flavor-changing neutral current.

Although our model is a simple toy model for the dynamical CP violation, it may be elaborated to fully account for the $\mathrm{CP}$ violation in the standard model. The investigation in this direction is in progress.

\section{ACKNOWLEDGEMENTS}

The authors would like to thank T. Onogi for discussions and suggestions and K. Yamawaki for useful informations. 


\section{REFERENCES}

1. M. Kobayashi and T. Maskawa, Prog. Theor. Phys. 49, 652 (1973).

2. X.-I. He, B. H. J. McKellar and S. Pakvasa, Int. J. Mod. Phys. A4, 5011 (1989).

3. L. S. Altarev et. al., Pis'ma Zh. Eksp. Teor. Fiz. 44, 360 (1986) (JETP Lett. 44, 460 (1986)).

K. Smith et. al., Phys. Lett. B234, 191 (1990).

4. S. Weinberg, Phys. Rev. Lett. 37, 657 (1976).

See also T. D. Lee, Phys. Rev. D8, 1226 (1973); Phys. Reports 9C, 143 (1974).

5. S. Weinberg, Phys. Rev. D13, 974 (1976); D19, 1277 (1978).

L. Susskind, Phys. Rev. D20, 2619 (1979).

6. Y. Nambu, Proc. Intern. Workshop on New Trends in Strong Coupling Gauge Theories, Nagoya, 1988, eds. M. Bando, T. Muta and K. Yamawaki (World Scientific Pub. Co., 1989).

V. A. Miransky, M. Tanabashi and K. Yamawaki, Phys. Lett. B211, 177 (1989); Mod. Phys. Lett. A4, 1043 (1989).

W. J. Marciano, Phys. Rev. Lett. 62, 2793 (1989); Phys. Rev. D41, 219 (1990).

W. A. Bardeen, C. T. Hill and M. Lindner, Phys. Rev. D41, 1647 (1990).

7. C. T. Hill, Phys. Lett. B266, 419 (1991).

8. C. T. Hill, M. A. Luty and E. A. Paschos, Phys. Rev. D43, 3011 (1991).

9. W. J. Marciano, Phys. Rev. D21, 2425 (1980).

K. Fukazawa, T. Muta, J. Saito, I. Watanabe, M. Yonezawa and M. Inoue, Prog. Theor. Phys. 85, 111 (1991).

10. R. Dashen, Phys. Rev. D3, 1879 (1971).

11. E. Eichten, K. Lane and J. Preskill, Phys. Rev. Lett. 45, 225 (1980).

12. W. Goldstein, Nucl. Phys. B213, 477 (1983); B229, 157 (1983).

13. R. N. Cahn and H. Harari, Nucl. Phys. B176, 135 (1980).

S. Dimopoulos and J. Ellis, Nucl. Phys. B182, 505 (1981). 